Canadian University Music Review

Revue de musique des universités canadiennes

\title{
Schumann ist der Dichter
}

\section{Henri Pousseur}

Numéro 2, 1981

URI : https://id.erudit.org/iderudit/1013746ar

DOI : https://doi.org/10.7202/1013746ar

Aller au sommaire du numéro

Éditeur(s)

Canadian University Music Society / Société de musique des universités

canadiennes

ISSN

0710-0353 (imprimé)

2291-2436 (numérique)

Découvrir la revue

Citer cet article

Pousseur, H. (1981). Schumann ist der Dichter. Canadian University Music Review / Revue de musique des universités canadiennes, (2), 94-113.

https://doi.org/10.7202/1013746ar

All Rights Reserved (C Canadian University Music Society / Société de musique des universités canadiennes, 1981
Ce document est protégé par la loi sur le droit d'auteur. L'utilisation des services d’Érudit (y compris la reproduction) est assujettie à sa politique d'utilisation que vous pouvez consulter en ligne.

https://apropos.erudit.org/fr/usagers/politique-dutilisation/ 


\title{
SCHUMANN IST DER DICHTER
}

\author{
Henri Pousseur
}

\section{Un cercle de fragments?}

Dans un numéro de L'Arc consacré à Beethoven, Roland Barthes (1970:15) distinguait la musique pratique (celle que l'on perçoit avec son corps agissant, par exemple avec ses mains, au clavier) de la musique seulement reçue, entendue, voire même écoutée. ${ }^{1}$ Il terminait cependant son texte en mettant en évidence une autre forme de pratique musicale, mode particulier de l'écoute, sur lequel, partageant tout à fait son penchant, je voudrais ici insister : c'est la lecture, et j'ajoute la lecture analytique, l'examen détaillé des structures et relations qui constituent une œuvre.

Mais attention! Il ne peut nullement s'agir d'une opération abstraite, appliquée à une matière morte ou du moins immobilisée, tuant celle-ci par ses interventions dissectrices. Au contraire, il s'agit, se faisant tout petit, à la fois humble et pourtant extraordinairement ambitieux (puisque décidé à presque égaler l'acte créateur) de pénétrer, comme Pinocchio dans le ventre de la baleine, au sein même de la musique en action, d'en observer de près, à l'aise, tous les mouvements, de s'attarder auprès de chacun d'eux, d'aller de l'un à l'autre, de déceler les coordinations à distance, de constater les stratégies d'ensemble auxquelles concourent les rouages partiels, pris toujours dans leur vivant fonctionnement.

L'oreille (intérieure) est donc ici continuellement à l'œuvre, mais c'est une oreille mobile, ubique, chercheuse, voire fouineuse. Ce type d'aperception, d'exploration à la fois amoureuse et vigilante, est particulièrement indiqué, par exemple, pour les ouvres de Webern, si vite passées alors que leur richesse exceptionnelle, leur densité d'information et de miroitement relationnel tout à fait unique, nécessite - et rend 
possibles - une présence et une confrontation bien plus prolongées!

Ce n'est certainement pas un hasard si Barthes, dans un autre de ses écrits (le fragment du Roland Barthes par Roland Barthes intitulé : "Le cercle des fragments »), avant d'affirmer : "Pièces brèves de Webern ... : quelle souveraineté il met à tourner court! » (1975: 98) (c'est évidemment du jeune Webern qu'il parle, alors que je viens de penser surtout à celui de la maturité), nomme dans un seul souffle ce musicien et un autre, séparé de lui par près d'un siècle : "L'homme qui a le mieux compris et pratiqué l'esthétique du fragment (avant Webern), c'est peut-être Schumann; ... tout ce qu'il produisait était finalement intercalé; mais entre quoi et quoi? Que veut dire une suite pure d'interruptions " (ibid.)?

Il avait déclaré avant cela : "Quoi, lorsqu'on met des fragments à la suite, nulle organisation possible? Si : le fragment est comme l'idée musicale d'un cycle (Bonne chanson, Dichterliebe) : chaque pièce se suffit, et cependant elle n'est jamais que l'interstice de ses voisines ..." (ibid.). Et plus haut encore, dès le début de ce fragment (lui-même extrêmement fragmenté, mais dont Barthes remarquera ensuite (1975:99), en une sorte d'auto-ironie intitulée "Le fragment comme illusion ": " mais comme le fragment ... est finalement un genre esthétique ..., en croyant me disperser, je ne fais que regagner sagement le lit de l'imaginaire ") : "Les fragments sont alors les pierres sur le pourtour du cercle : ... au centre, quoi » (1975:96)?

Prenons donc Dichterliebe, insinuons-nous dans les fissures entre les pièces (ou entre leurs éléments); efforçonsnous d'éprouver la qualité, le sens de ces silences (et gardons la tête suffisamment froide pour en évaluer les articulations descriptibles); rappelons-nous que si chez Webern la grande discontinuité (entre tous les sons) est un facteur capital d'affranchissement par rapport à l'illusion classique de continuité linéaire, cette discontinuité n'empêche nullement, permet au contraire (pour avoir révélé leurs éléments) la constitution de 
figures constellées, manifestant ainsi un autre, plus profond et plus essentiel niveau de solidarité (comme le fait remarquer quelque part Michel Butor qui insistait récemment sur l'importance pour nous des ouvrages en forme de recueils, mais en fixant surtout notre regard sur leur organisation : n'a-t-il pas fait apparaître les propriétés les plus fortement structurées de ces mystérieuses "Variations Diabelli » que nous ne pourrons plus jamais, après cela, entendre comme avant)?

Tâchons même, à force d'insistance investigatrice, d'atteindre le centre du cercle que l'œuvre de Schumann semble bien constituer (il s'agit, nous le verrons tout de suite, d'un cycle au sens le plus complet) : peut-être aurons-nous finalement la chance de trouver quelques bribes d'une réponse aux questions posées?

\section{Désir d'amour ${ }^{2}$}

La première pièce, et avec elle tout le cycle, commence immédiatement par la dissonance la plus tendue, dans une disposition pour un bref moment littéralement wébernienne; au do dièse de la main droite vient s'ajouter un ré à la main gauche, deux octaves plus bas.

Certes, ce qui fait suite à ce dernier, l'arpège montant de l'accord de sixte de si mineur, avec appoggiature chromatique inférieure du fondamental (la dièse, d'ailleurs harmonisé très fugitivement au do dièse dont il n'attendra cependant pas la résolution), va tout de suite donner à cette tension son sens tonal ; c'est bien d'un phénomène appellatif qu'il s'agit : do dièse, dont nous aurons encore l'occasion de découvrir l'importance dominante pour toute l'œuvre, apparaît alors comme retard du si (on aurait peut-être tendance à le considérer plutôt comme une appoggiature, mais en fait, il est préparé : par sa présence initiale isolée, qui définit tout de suite sa fonction primordiale, au moins pour cette pièce).

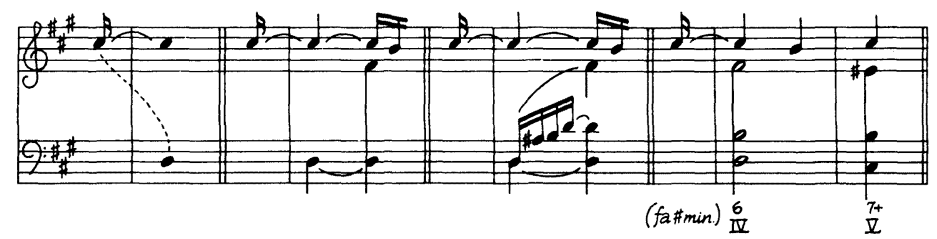


Ce premier mouvement d'attraction résolutive n'est que la manifestation, au niveau du détail, de trois niveaux d'appel hiérarchisés : le ré de la basse (tierce de l'accord de si), descend, une mesure plus tard, grâce à un mouvement de demi-ton (typique de l'expression de la plainte et qui "rime " d'ailleurs avec la seconde descendante do dièse-si du dessus), à do dièse dont l'arpège s'avérera incontestablement être un accord de dominante: tierce majeure (mi dièse) et septième (si) ceci qualifie, sans équivoque possible, l'accord précédent comme accord de sous-dominante, antépénultième caractéristique, qui fait désirer avec une extrême véhémence (c'est le deuxième niveau) son passage à l'interrogation définitive.

Quant à celle-ci, nul n'ignore qu'elle devrait se résoudre sur la tonique (dont nous savons par anticipation conventionnelle qu'il s'agit de fa dièse, probablement mineur, ce que confirme d'ailleurs l'« armure » de la pièce, c'est-à-dire les troís dièse écrits "à la clé »); elle reste cependant suspendue; l'enchaînement ré-do dièse à la basse (et avec lui, à quelques détails près, la polyphonie que celle-ci supporte) est immédiatement répété, terminant ce petit prélude de piano et amenant la phrase chantée, qui ne fera pas non plus entendre l'accord de tonique, puisqu'elle modulera immédiatement à une ou plusieurs tonalités différentes. Une simple lecture du morceau nous révèle d'ailleurs que ces deux périodes (piano seul et chant accompagné) alterneront selon la formule ABABA (avec de nouvelles légères variantes, significatives mais négligeables pour notre propos, en particulier dans la basse de A). La pièce se terminera donc par un suspens de l'accord de septième de dominante non-résolu, la tonique du morceau (c'est-à-dire celle des parties de piano qui l'encadrent) n'étant pas présentée une seule fois sous forme d'accord. C'est là un artifice bien schumannien, mais unique dans son extrémité (c'est aussi le troisième niveau) et présent une seule fois dans ce cycle, donnent au premier morceau son caractère de prélude interrogatif.

Avant d'examiner la phrase avec voix, remarquons encore le développement mélodique de la main droite dans la ritournelle de piano: la descente do dièse-si dont nous avons vu le sens harmonique, sert de tremplin pour un saut montant de sixte majeure, suivi d'une retombée de deux secondes, l'une majeure et l'autre mineure (sol dièse, fa dièse, mi dièse), formant un intervalle total de tierce mineure, elle-même renversement (dans un sens double puisqu'elle descend) de la sixte qu'on 
vient d'entendre, et s'harmonisant d'ailleurs au mouvement descendant de la basse :

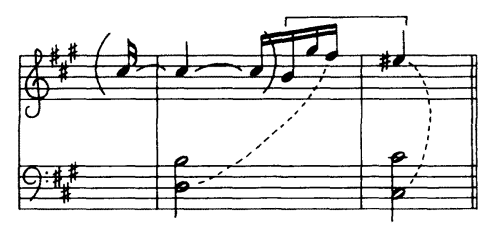

Exemple 2

Lors de sa reprise (immédiate), cette formule mélodique expansive sera précédée d'un autre bref motif qui ramène le do dièse tenu.

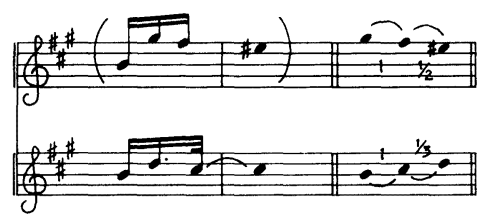

Exemple 3

Défini lui aussi par une extension totale de tierce mineure (dont les intervalles internes, secondes majeure et mineure, sont cependant en ordre vertical inversé, la plus petite au-dessus), il donne au contenu de celle-ci une courbe mélodique différente, brisée, rappelant en plus petit celle du motif précédent (grand mouvement ascendant et plus petit mouvement descendant) et anticipant une figure qui sera pour toute l'œuvre d'une signification déterminante.

La mélodie du chant, dans sa première phrase, apparaît tout de suite comme une variante complexe, et extraordinairement subtile, de celle du piano: 


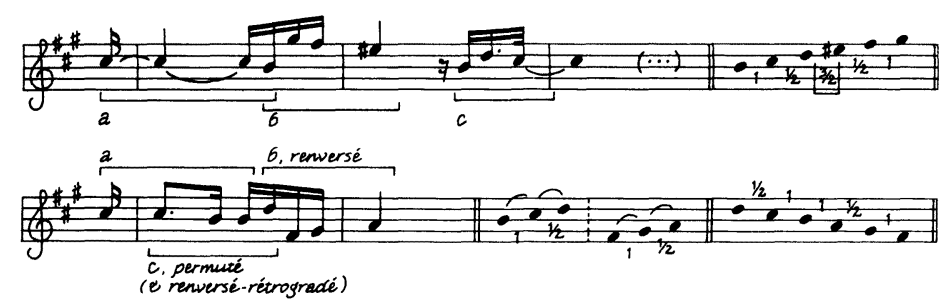

Exemple 4

Si on supprime la deuxième note, si (articulée deux fois par le texte), il s'agit d'un renversement presque exact du motif de sixte : à ceci près que la sixte est mineure, et non plus majeure. La seconde qui la précède est par conséquent mineure elle aussi, tous les autres intervalles (y compris ceux du do dièse initial aux notes finales) étant exactement conservés.

Mais le si ajouté transforme également la première seconde montante (qu'il sépare) en une variante du motif de tierce, à la fois permutation de ses notes constitutives et renversement rétrogradé de son contour, avec variation fine des intervalles (la seconde immédiate qui était mineure, est ici majeure tandis qu'une autre seconde, cachée entre la première et la troisième note, subit évidemment la variation inverse). Cette fusion plus intime des deux motifs dans lesquels se subdivisait la phrase du piano, coïncide avec une disparition de la césure et avec le rassemblement plus serré, en une seule unité continue, de la séquence chantée (cependant toujours articulée en une note longue - chargée d'énergie motrice contenue - et un mouvement rapide dans lequel cette accumulation se décharge). Ceci met particulièrement en évidence le rapport des deux groupes de tierce mineure (si-ré, sol dièse-mi dièse d'une part, rapport de triton, mouvement et ordre vertical des secondes inversés; si-ré, fa dièse-la de l'autre, rapport de quarte juste, mouvement et ordre des secondes droits, simplement transposés).

Cette phrase est " accompagnée " au piano (en une polyphonie dont nous ne pouvons qu'admirer l'articulation aussi savante que raffinée, développement peu banal des arpèges du début, eux-mêmes déjà si subtilement ornés) par une harmonisation en la majeur: 


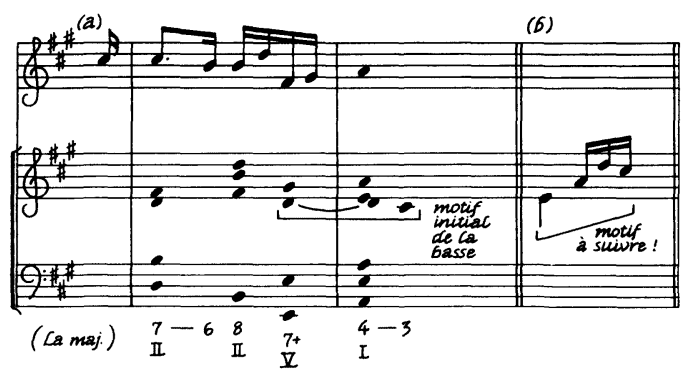

Exemple 5

L'accord de sixte de si mineur, conservé, et dont notre do dièse apparaît encore comme un retard, est maintenant interprété comme deuxième degré, substitut de la sous-dominante (donc quasi-dominante de la dominante, fonction toujours typiquement antépénultième). Il amène effectivement l'accord de septième de dominante qui se résoudra normalement sur sa tonique, chaque accord n'occupant plus qu'une noire ou demi mesure, ce qui, par rapport à la période de piano solo, accélère sensiblement le rythme harmonique (il faut faire partiellement exception pour le dernier, l'accord de tonique, qui occupe à nouveau toute la mesure, mais dont la deuxième partie est articulée de manière à préparer la suite et amener une nouvelle harmonie).

Après une reprise de cette première phrase chantée qui donne déjà à la partie de chant une longueur (et une structure répétitive) égale à celle de la phrase du piano (quatre mesures), nous assistons cependant encore à l'adjonction de quatre mesures supplémentaires, toujours groupées deux par deux et répétitions l'une de l'autre.

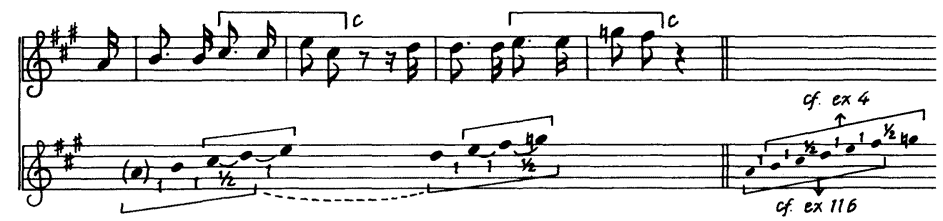

Exemple 6

Mais cette fois, il y a transposition et progression harmonique ascendante, ce qui augmente sensiblement l'impatience qu'elle exprime (si la première phrase dont les deux expositions correspondent à deux vers du poème, parle du 
" merveilleux mois de mai » et de ses signes caractéristiques : éclosion des bourgeons et chant des oiseaux, la deuxième décrit les pulsions amoureuses éveillées par cette poussée vitale).

Harmoniquement, nous avons maintenant à faire aux tonalités successives de si mineur et de ré majeur (mais avec une coloration particulière que nous allons examiner) et nous pouvons dès à présent constater, en feuilletant quelques pages subséquentes du recueil, que les trois tonalités (passagères, subordonnées) de la strophe chantée (dont nous constatons aussi que la durée s'accélère : quatre mesures en la, et deux mesures respectivement en si et en ré, autre expression de l'impatience déjà décrite) anticipent sur les tonalités constitutives de trois morceaux immédiatement ou presque immédiatement suivants, soit, dans l'ordre, le deuxième, le cinquième et le troisième.

La structure harmonique interne de chacun des éléments de deux mesures (évidemment identiques - à part certains éléments modaux - puisqu'il y a progression) reprend la structure à trois " temps " dont nous avons déjà rencontré deux versions : antépénultième, interrogation principale ou "dominante ", et résolution de celle-ci. De la première version (qui omettait le troisième temps et nourrissait ainsi notre insatisfaction et notre nostalgie), elle reprend le mouvement mélodique de la basse, soit un demi-ton descendant (ce qui donne précisément la couleur particulière de la section en ré majeur, puisque, pour obtenir ce demi-ton, son sixième degré se trouve baissé : si bémol, enharmonie du la dièse entendu dans les mesures précédentes et présent dès la ritournelle - et que sa sousdominante, dès lors, se trouve "minorisée ») :

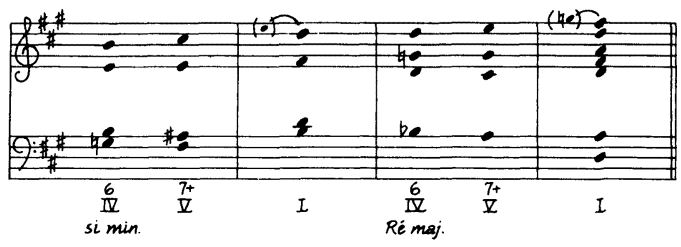

Exemple 7

Mais cet intervalle est maintenant suivi, soit d'une quarte montante, soit de son renversement, une quinte descendante. C'est donc la deuxième version qui fournit cet intervalle (là, il était enrobé par le mouvement de doubles croches, dans lequel 
nous mettrons encore en évidence, anticipation d'un futur motif important, les deux quartes montantes suivies d'un demi-ton descendant (cf. l'exemple 5 b : ils servent ici de "pont " vers la reprise ou vers la phrase suivante, tout en incluant une variante, un renversement rétrogradé du mouvement de basse que nous venons de signaler et dont nous rencontrerons encore d'autres expositions, plus ou moins textuelles). Autrement dit, la nouvelle structure reprend la séquence ternaire, complète et résolutive.

Elle fait en outre percevoir la parenté par assimilation des formules se terminant toutes par une quinte - ou quarte contraire - mais commençant soit par un demi-ton descendant, soit par un ton ou même une quarte de sens contraire, inauguration de tout un système d'apparentements entre les motifs superficiellement les plus diversifiés. Elle reprend enfin au second modèle son rythme d'accords accéléré, dont nous avons déjà vu qu'elle le conjuguait avec une accélération du rythme tonal et accroissait ainsi son dynamisme :

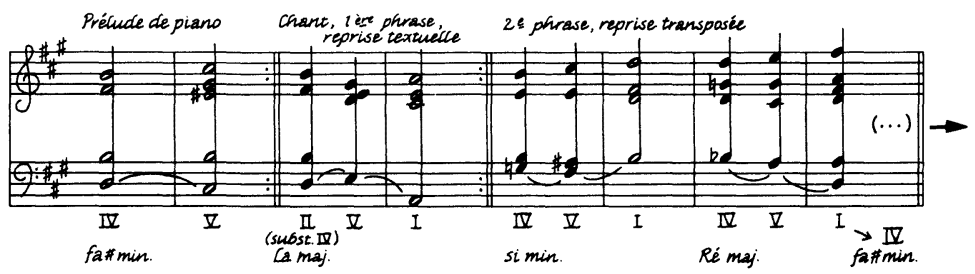

Exemple 8

Au chant, la situation n'est pas moins remarquable. Etalé en valeurs plus longues, nous retrouvons le motif de tierce mineure montante (précédé maintenant d'une seconde majeure de même sens) et de seconde descendante, majeure ou mineure selon le mode de la période. C'est le motif typique de la respiration, soupir ou halètement selon son rythme, mais toujours coloré de désir du fait de sa direction montante, ellemême multipliée par la répétition transposée, de nouveau à la tierce mineure. Cette nouvelle phrase conduit à la reprise du " refrain » de piano, et nous remarquons le rapprochement qui en résulte, au même registre - du moins au même registre écrit, la voix étant d'ailleurs doublée par le piano, et même à la limite supérieure de la tessiture, soit en posture d'extrême exposition des secondes sol bécarre-fa dièse (précédée de mi bécarre) et sol dièse-fa dièse (suivie de mi dièse) : 


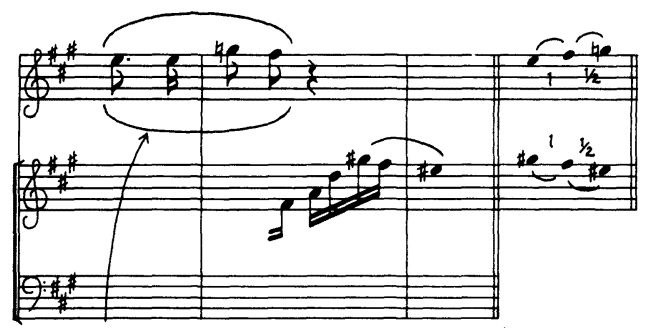

ceci étant une transposition du motif $c$ du prélude de piano,

Ce rapprochement effectué dans notre exemple 3

est ici rendu perticulièrement évident.

\section{Exemple 9}

Rapprochement d'autant plus serré qu'il y a agglutination de la dernière mesure de l'une et de la première mesure de l'autre période (vu le ré commun à la basse), et donc suppression d'une mesure des deuxième et troisième versions de la ritournelle du piano :

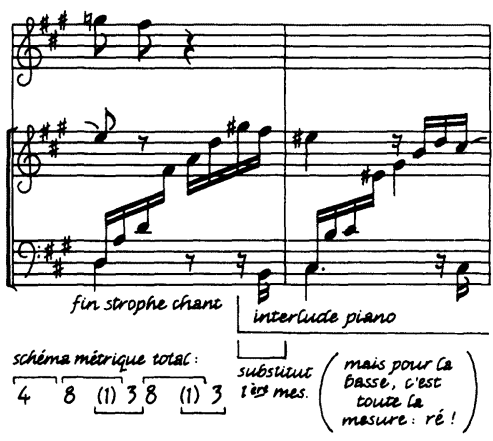

Exemple 10

Enfin, un dernier coup d'œil panoramique sur l'ensemble de la pièce nous révèle un premier véritable isomorphisme entre niveaux très différents, celui du détail mélodique et celui du plan total: si nous considérons les toniques des différentes tonalités partielles (donc fa dièse pour les sections de piano), nous obtenons l'exemple 11a, soit les notes autour desquelles s'articule la première phrase de la voix iqui y ajoute seulement deux notes intermédiaires, une à l'intérieur de chaque tierce) ; mais si nous considérons les fondamentaux des accords sur lesquels les différentes périodes tonales se terminent (mise en évidence dont nous trouverons encore plus tard une justification particulièrement significative), nous obtenons l'exemple $11 \mathrm{~b}$, 
soit un déploiement répétitif de notre motif de tierce, exprimé par les notes réelles de sa première apparition au piano (auquel est ajouté la, note vers laquelle il tend lors de sa prochaine apparition, permutée, à la voix) ; c'est aussi la transposition exacte, à la quarte inférieure de son dernier et plus expressif déploiement (en ré majeur), qui supporte les paroles les plus passionnées, annonçant l'éclosion de l'amour (" die Liebe aufgegangen ») et donannt voix à son désir impétueux (" mein Sehnen und Verlangen »).

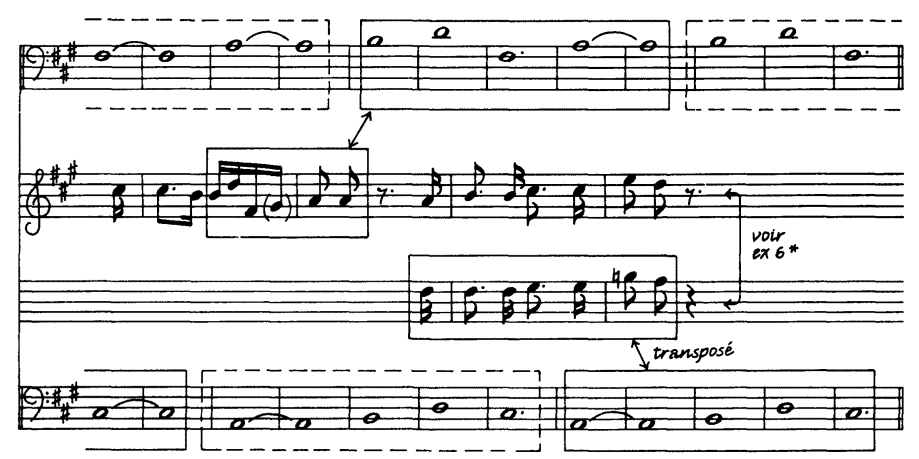

Exemple 11a

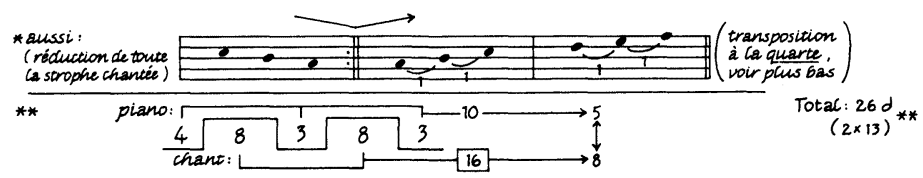

Exemple 11b

\section{Presque une série}

Les constatations que nous venons de faire devraient avoir suscité notre interrogation la plus vive : cet état de choses va-t-il se répéter tout au long du cycle? Et surtout : retrouveronsnous quelque chose d'analogue au niveau total? Et quel sera alors son sens (tant poétique qu'historique)?

Nous sommes tout de suite amenés à examiner une première expression de cette structure globale, dans un segment de laquelle nous nous sommes d'ailleurs déjà laissés entraîner, et dont l'organisation systématique saute assez aisément aux yeux: chacune des seize pièces qui constituent le cycle est 
située dans une tonalité différente ou, à défaut de différence de tonique, dans un mode différent, majeur ou mineur (il n'y a donc que deux pièces, au plus, ayant la même tonique).

Mieux, à une exception près (entre la douzième et la treizième pièce), il y a, d'une pièce à la suivante, au plus une différence d'altérations constitutives ("armure » ou nombre d'altérations à la clé).

S'il n'y a pas de différence, c'est qu'on passe à la " tonalité relative ", majeure ou mineure (par exemple, de la première à la deuxième pièce, on passe de fa dièse mineur à la majeur, les deux tonalités ayant trois dièses à la clé - et ce changement, nous le savons déjà, ne fait que reproduire ce qui s'est passé à l'intérieur de la première pièce); les toniques sont alors à distance de tierce mineure.

S'il y a changement, c'est qu'on passe

- soit à la dominante ou à la sous-dominante de la tonalité précédente, sans changer de mode (tonique à la quinte supérieure ou inférieure, une seule altération de différence : par exemple, de la deuxième à la troisième pièce, respectivement en la et ré majeur, on " perd » un dièse),

- soit au "relatif " de l'une de ces deux tonalités (on change donc de mode et de tonalité, et les toniques sont alors toujours - du moins à l'intérieur de ce cycle - à distance de tierce majeure : par exemple, de sol majeur à si mineur, pièces IV à $\mathrm{V}$, ou de mi mineur à do majeur, pièces VI à VII).

Enfin, la suite de ces changements, qui constitue le mouvement modulatoire d'ensemble de l'œuvre (et est seulement contrecarré dans son orientation principale, de manière momentanée, par quelques légers mouvements en sens contraire) s'effectue "vers la gauche " (dans le "cycle des quintes " que l'on écrit conventionnellement de cette manière), c'est-à-dire vers des tonalités ayant de moins en moins de dièses et, ensuite, de plus en plus de bémols. La réapparition des dièses à la quatorzième pièce n'est à première vue qu'un artifice " enharmonique » pour continuer dans le même sens général; mais on sait que vu le caractère circulaire du matériau chromatique tempéré, ce mouvement unidirectionnel nous ramène finalement vers notre point de départ (la dernière pièce est - ou du moins commence - en do dièse mineur, avec quatre 
dièses à la clé, et est donc par rapport à la première dans une situation de voisinage tonal conforme à l'une des possibilités définies plus haut et qui caractérisent toute l'œuvre). Nous verrons encore que non seulement cette circularité, mais même l'apparition de l'enharmonie à un point bien précis du parcours (et sa réapparition à l'extrême fin de celui-ci, pour le postlude instrumental de la seizième pièce - en ré bémol majeur) sont loin d'être dépourvus de signification!

Voici maintenant une première représentation résumée du cycle où chaque pièce est représentée par sa tonique (notes noires), pourvue de sa tierce supérieure (notes blanches) afin de montrer s'il s'agit d'un mode majeur ou mineur (les altérations ne valent que pour la note qu'elles précèdent) :

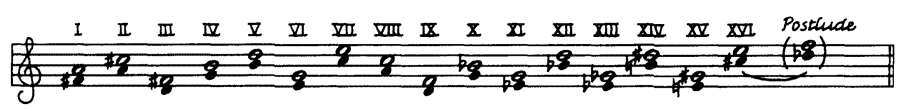

Exemple 12

Nous constatons tout de suite que les différentes possibilités de relation tonale d'une pièce à l'autre sont distribuées de manière assez complexe et que la circularité et la progressivité presque totale du mouvement n'empêchent nullement l'existence d'une forme originale et intéressante. Des considérations analytiques supplémentaires vont encore nous faire découvrir d'autres aspects de sa richesse véritablement génératrice.

Commençons par séparer les tonalités majeures des tonalités mineures :

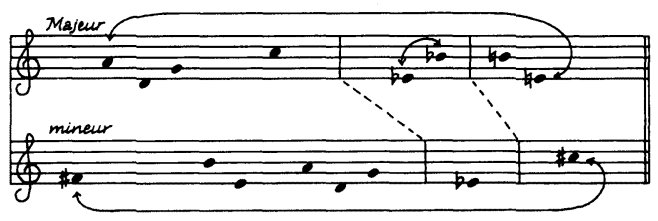


Nous constatons qu'il y a un nombre égal de tonalités majeures et mineures (huit de chaque mode), si l'on fait abstraction du postlude pour piano seul de la seizième pièce (on avait en ce temps l'habitude - sans doute significative de qualifier les morceaux par leur tonalité de départ et non d'arrivée; exemples : les cinquième et neuvième symphonies de Beethoven). Nous constatons aussi que dans chaque " couche" modale, à part une inversion simple ( $\mathrm{mi}$ bémol et si bémol majeurs) et quelques omissions (huit au total, évidemment), nous avons à faire au déploiement régulier du cycle des quintes, tournart "vers la gauche " (c'est-à-dire vers le bas si l'on tient compte du fait qu'une quinte a un fondamental qui est précisément, dans cette représentation, sa note de gauche). Toutefois, du fait de la circularité, nous nous retrouvons, à la fin de l'œuvre, immédiatement "à droite " de notre point de départ, autrement dit, cette longue descente nous a permis de monter d'un degré.

Nous constatons enfin que les interruptions et interpolations des deux versions du cycle des quintes se font elles aussi selon une stratégie remarquable dans laquelle nous pouvons déjà faire apparaître quelques points forts :

a) "groupes » de une, deux ou trois pièces de même mode, séparés les uns des autres par les groupes du mode inverse ;

b) si on associe la dernière et la première pièce, la répartition (hors-temps de ces groupes est la même pour les deux modes : 3-1-2-2 pour le majeur et 2-(1+1)-3-1-2 pour le mineur (c'est vrai m̧ême pour les omissions : 1-3 et 3-1);

c) non seulement les intervalles sont évidemment les mêmes (toujours des quintes), mais aussi une grande partie des notes : nous retrouvons des deux côtés les groupes ternaires la-ré-sol, et les groupes binaires simi (du fait de la circularité, leur ordre dans le temps est inversé); le couple mi bémol-si bémol de la couche majeure correspond encore au mi bémol isolé de la couche mineure (qui lui fait d'ailleurs immédiatement suite), tandis que les trois notes restantes sont sans correspondance;

d) c'est dire qu'il y a, même dans les omissions, certaines symétries : fa et la bémol sont absents des deux côtés, 
tandis que viennent s'ajouter à elles, de chaque côté, deux notes différentes, ce qui fait un total de six:

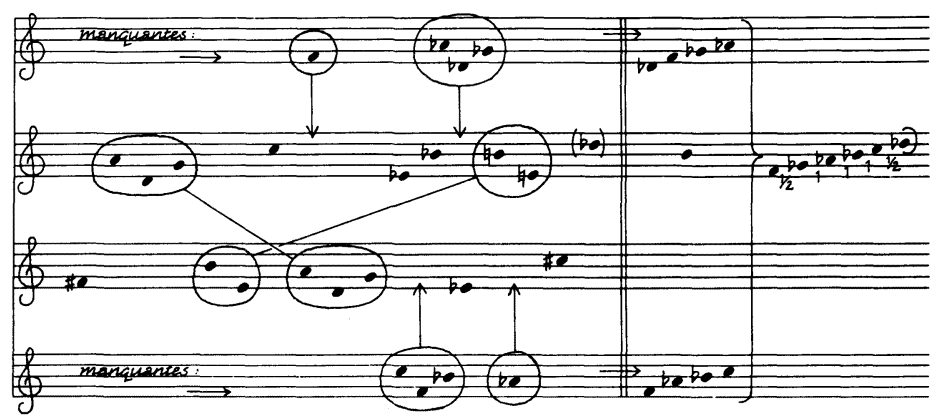

Exemple 14

Et nous découvrons, en les groupant par ordre d'échelle (à droite de l'exemple), qu'il s'agit d'un hexacorde de ré bémol majeur, soit de la tonalité qui terminera l'œuvre et qui, à ce niveau, se trouve ainsi en quelque sorte économisée, mise en réserve.

Reprenons encore une fois la séquence totale :

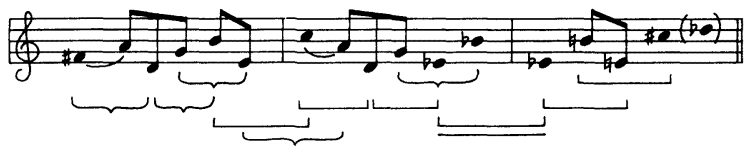

Exemple 15

Les deux groupes de trois sont encadrés de manière très symétrique: le premier est précédé d'une tierce mineure montante, le second, d'une tierce mineure descendante; le premier est suivi d'une tierce majeure montante et d'une quinte descendante (accord parfait mineur), le second, des mêmes intervalles, de nouveau en sens inverse (accord parfait majeur). Ceci semble nous autoriser à distinguer ces deux groupes de six comme des entités indépendantes. Reste le dernier groupe de quatre! 
Remarquons que si les deux quintes successives du premier groupe (la-ré-sol) forment avec l'intervalle qui leur est immédiatement voisin (précédent ou suivant) une même figure (accord parfait majeur, toujours représenté par une accolade), celles du deuxième groupe forment des ensembles légèrement plus «dissonants", et moins similaires : tierce mineure et quinte de même sens formant une septième mineure, tandis que quinte et tierce majeure forment une septième majeure (crochets simples sous la portée). Or, la quinte centrale du groupe final de quatre qui est identique à la quinte finale du premier groupe (si-mi), est précisément encadrée d'une tierce majeure et d'une tierce mineure de même sens, ce qui veut dire qu'elle réalise, condensés et dans l'ordre inverse, les deux ensembles caractéristiques issus du deuxième groupe de trois. On pourrait donc considérer le groupe final comme une synthèse des deux premiers groupes.

Nous constatons encore que le début de ce troisième groupe est marqué par le changement d'armure (et donc de climat harmonique) le plus important, accusé par le fait qu'il a été précédé par un petit mouvement en sens contraire (après être passé de trois à deux bémols, on en trouve soudain six) ; mais aussi cette rupture est fortement compensée par le rapport entre les toniques ( $\mathrm{mi}$ bémol majeur-si bémol majeur-mi bémol mineur : cadence $\mathrm{I}_{M}-\mathrm{V}-\mathrm{I}_{\mathrm{m}}$ ).

C'est le seul endroit de la séquence où nous trouvions (entre toniques) un unisson aussi rapproché et où un intervalle soit simplement l'inverse du précédent (crochet double). Tous les autres groupes de trois notes successives, même aux " joints" entre les différentes parties (ou entre la fin et le début!) appartiennent aux catégories déjà mentionnées : accords parfaits, majeurs ou mineurs, accords de septième, majeure ou mineure (accolades ou crochets en pointillés).

De même, la séparation entre les deux premiers groupes correspond (est-ce hasard?) à la disparition des tonalités à dièse (sauf celles de la fin, apparaissant précisément après l'enharmonie) et à l'apparition de do majeur, tonalité semblant affectivement très chargée aux oreilles de Schumann.

La division en trois parties que nous venons de mettre en évidence, paraît se justifier encore à (au moins) deux autres 
points de vue. D'abord, du point de vue proportionnel, considéré à ce niveau très global des nombres de pièces par " partie ». Si nous simplifions, divisons par deux ces nombres (tous pairs), nous voyons que la structure d'ensemble est régie par le souci de se rapprocher d'une progression par "nombre d'or », qu'elle est conforme à certains termes d'une série de

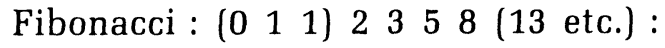

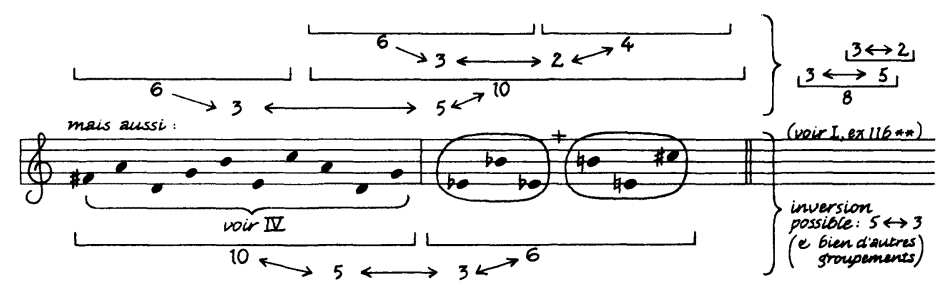

Exemple 16

Le fait que la pièce dont l'apparition se produit de la manière la moins normale (plus grand changement d'armure, à quoi s'ajoutent, nous le verrons encore, d'autres facteurs extrêmes de mise en évidence) soit justement la treizième (cette fois, sans simplification), vient encore corroborer ce souci, qui ne devrait pas trop nous étonner de la part de Schumann. Nous verrons plus bas que ce premier niveau de pertinence est modulé d'une façon extraordinairement raffinée aux niveaux inférieurs; par exemple au niveau des nombres de vers par poème où la structure initiale de longueur des parties se trouve renversée, d'une manière parfaitement régulière et significative.

Mais le point de vue où la division (ou plutôt l'articulation) s'impose avec le maximum de légitimité, c'est le point de vue poétique, narratif. En effet, même si les premières six pièces, qui parlent de l'éveil de l'amour et de ses premiers emportements, ne sont pas entièrement dénuées de tristesse, du moins n'y découvrons-nous, à première vue, aucun motif " sérieux » à celle-ci. Il y a bien une certaine tendance dépressive encore assez discrète, qui se dessine au cours de ce premier groupe. Mise à part la relative inquiétude (ou nostalgie) exprimée par la ritournelle du piano dans la première pièce (et qui lui donne son caractère introductif, annonciateur), les quatre premiers lieder sont certainement les plus heureux. Nous entendons un sanglot, d'abord inexplicable, à la fin du quatrième (de sa partie chantée), et les deux pièces qui suivent, de nouveau mineures, 
en subissent la coloration : la fébrilité de l'une, la gravité de l'autre accusent toutes deux la prémonition d'une perte, exprimée par le passé dans lequel sont décrites les joies amoureuses, soit littéralement (den sie mir einst gegeben), soit du fait que leur objet se trouve hypostasié, figé sous les traits d'une figure très ancienne. (Qu'il y ait à nouveau une certaine subdivision et organisation à l'intérieur de chacune des grandes sections est d'ailleurs bien normal, et nous verrons encore que la quatrième pièce, symétrique de la treizième, joue dans l'ensemble un rôle très particulier.)

Mais quelle différence tout de même quand éclate, au célèbre septième lied (et en do majeur!) l'orage des reproches! Même réprimés (ich grolle nicht...), comme ils sont éloquents! Toute la deuxième partie (selon la division que nous venons de proposer) sera consacrée à décrire la cause du profond déchirement qui est au centre de l'histoire contée : l'aimée a quitté le narrateur, lui a préféré un autre, et le premier est plongé dans un inconsolable chagrin.

Le point extrême de cette descente aux enfers sera sans aucun doute la fameuse treizième pièce; il se situera donc immédiatement après la fin de notre deuxième division (illustrant peut-être le fait qu'en analyse musicale, il faut moins découper que montrer les joints, les charnières, les éléments communs à deux phrases ou parties, leur permettant de jouer l'une par rapport à l'autre). Mais ce qui nous permet de considérer tout de même cette treizième pièce avant tout comme le début du chapitre final, c'est, outre les éléments distinctifs formels déjà mentionnés, l'apparition d'un thème nouveau, celui du rêve, commun aux trois morceaux qui s'enchaînent à partir d'ici.

Ce rêve est d'abord ùn cauchemar, vécu au plus profond des ténèbres du cœur. Mais peu à peu, en passant d'une pièce à l'autre, il va s'éclaircir, ou du moins se tempérer d'oubli, puis d'indifférence; il va amener, rendre légitime le mouvement final de guérison, le rejet ou dépassement volontaire de la douleur. La circularité théorique du matériau sonore s'accompagne donc, grâce aux puissances imaginaires et nocturnes, d'un renversement thématique, d'une véritable remontée dont nous aurons encore l'occasion d'examiner les modalités les plus expressives. 
Dernier argument en faveur de notre partition de l'ensemble : en groupant les toniques de chacune des parties selon leur ordre scalaire, nous trouvons chaque fois un fragment de gamme, hexacorde ou tétracorde, ce qui ne serait certainement le cas avec aucune autre division par quatre (les divisions par six qui répondraient à cette condition se trouvent à l'intersection des deux premières parties; leur prise en considération - intéressante - ne nous apporterait cependant rien de plus " utile » pour notre propos) :

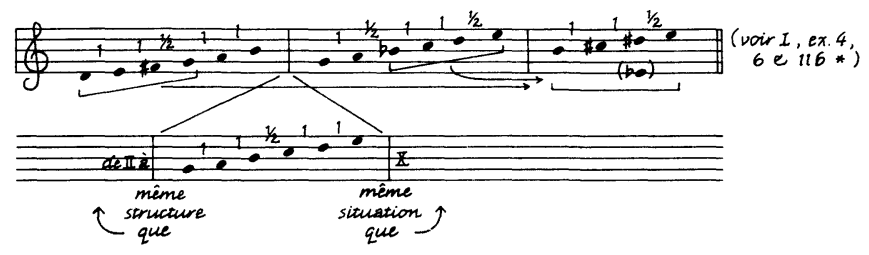

Exemple 17

Les tonalités ainsi définies sont bien celles qui dominent les trois parties : sol majeur, sol mineur et mi majeur (remarquons tout de même le do majeur prolongé de l'intersection!) soit trois tonalités formant, du point de vue des changements d'armure, une sorte de triangle isocèle : $\operatorname{sol} M$ à sol $\mathrm{m}: 3$; sol m à mi $\mathrm{M}: 6 ; \mathrm{mi} \mathrm{M}$ à sol $\mathrm{M}$ : à nouveau trois altérations "vers la gauche ". Ici aussi, les deuxième et troisième parties sont les plus distantes l'une de l'autre. Et le passage, de majeur à mineur et retour (qui sera encore considérablement raffiné au niveau du mouvement réel), correspond bien à l'évolution narrative que nous venons de résumer.

Enfin, ces fragments de gammes (dont nous avions déjà trouvé un exemple au niveau des notes omises) sont caractéristiques des structures mélodique de l'ouvre qui s'appuient très souvent sur des échelles tétra- ou hexacordiques. Mais nous pouvons aller plus loin: la manière dont les notes de ces échelles sont permutées, ordonnées " en temps réel » de manière à produire des "lignes brisées " d'une extrême sensibilité, est la même à ce niveau profond des rapports de tonalité, qu'au niveau des démarches mélodiques, soit dans leurs tournures les plus exposées et les plus représentatives, soit dans la définition de leur ossature ou charpente, c'est-à-dire de la série de leurs points principaux (il serait d'ailleurs possible de trouver ici quelques-uns des traits distinctifs de la mélodique schumannienne, 
opposée par exemple à celle d'un Schubert ou d'un Mozart : les "lignes " de ces derniers sont beaucoup plus basées, ou sur les arpèges, ou sur les gammes, véritablement conjointes et unidirectionnelles).

\section{NOTES}

1. Ce texte constitue le premier chapitre d'un ouvrage encore inédit, à paraître en allemand sous le titre Schumann ist der Dichter, Münich, Edition text + kritik, série Musik-konzepte.

2. En voici la traduction:

Au merveilleux mois de mai,

Quand tous les bourgeons s'ouvraient,

Voilà que dans mon cœur

A éclaté l'amour.

Au merveilleux mois de mai,

Quand tous les oiseaux chantaient,

Je lui ai avoué

Mes ardeurs et mon désir.

Pour ce Lied (en fa dièse mineur, à 214 à la noire, précédé de la mention : Langsamzart, lent, tendre et délicat), on se reportera à une édition courante des Dichterliebe.

\section{RÉFÉRENCES}

BARTHES, R.

1970 : «Musica practica », L'Arc, $\mathrm{N}^{\circ} 40,15-17$.

1975 : Roland Barthes par Roland Barthes. Paris : Seuil. 